\title{
Development and validation of bexarote by bioanalytical methods using liquid chromatography-tandem mass spectroscopy (LC-MS/MS)
}

\author{
K. Nagaraju ${ }^{1,2}$, Y. A. Chowdary ${ }^{3^{*}}$ and M. V. Basaveswara Rao ${ }^{4}$
}

\begin{abstract}
Background: The aim of this study was to develop and validate accurate and precise UPLC method with tandem mass spectrometry (Waters) for the determination of bexarotene in human plasma using bexarotene D4 as internal standard (IS).

Results: The retention time of bexarotene was $2.75 \pm 0.30 \mathrm{~min}$. The method was validated with respect to system suitability, linearity, accuracy, precision, matrix effect, auto sampler carryover test, and recovery. Linearity was found to be 1.04 to $351.93 \mathrm{\mu g} / \mathrm{mL}$. LOQQC, LQC, INTQC, MQC, and HQC were found to be 1.0550, 2.7800, 25.2700, 131.61, and 263.23 respectively. The mean percentage recovery was found to be $95.72 \%$

Conclusion: The bioanalytical method, a selective and sensitive liquid chromatography-mass spectrometry method to quantitate bexarotene in $\mathrm{K}_{2}$ EDTA human plasma over the concentration range 1.0440 to $351.9320 \mathrm{ng} / \mathrm{mL}$, was successfully validated. This method is suitable for sample analysis to support bioequivalence/bioavailability and/or pharmacokinetic studies involving formulations of bexarotene.
\end{abstract}

Keywords: Bexarotene, Validation, Liquid chromatography, Mass spectrometer

\section{Background}

Bexarotene (brand name: Targretin) [1] is an antineoplastic (anticancer) agent approved by the US Food and Drug Administration (FDA) (in late 1999) and the European Medicines Agency (EMA) (early 2001) for use as a treatment for cutaneous T cell lymphoma (CTCL) (Fig. 1) [2, 3]. It is a third-generation retinoid. The retinoic acid receptors (RARs) regulate cell differentiation and proliferation whereas RXRs regulate apoptosis [4]. LC-MS-based method that utilized both RPLC and HILIC separations was carried out [1-4], followed by multivariate data analysis to discriminate the global urine profiles of $\mathrm{BC}$ patients and healthy controls $[1,5]$. The purpose of this study was to identify a potential

\footnotetext{
* Correspondence: yarlagaddaac@yahoo.co.in

${ }^{3} \mathrm{NRI}$ College of Pharmacy, Pothavarappadu, Agiripalli, Rural, Krishna District, Vijayawada, AP, India

Full list of author information is available at the end of the article
}

biomarker pattern in urine using metabonomics to aid noninvasive $\mathrm{BC}$ detection using complementary chromatographic techniques $[6,7]$.

\section{Methods}

A few methods are available in literature [3, 8-16]. A new bioanalytical LC-MS/MS method was performed on the LC-MS/MS (API 4000) [6, 7, 17], consisting of binary gradient pump UV detector (LC-20 AD) employed for analysis, and rheodyne injector with $20 \mu \mathrm{l}$ fixed loop was used for the present study. Bexarotene was eluted with a flow rate of $1 \mathrm{ml} / \mathrm{min}$ using a mobile phase of acetonitrile: buffer $1(90: 10, \mathrm{v} / \mathrm{v})$. The retention time of bexarotene analyte is $2.75 \pm 0.3 \mathrm{~min}$.

\section{Springer Open}

(-) The Author(s). 2021 Open Access This article is licensed under a Creative Commons Attribution 4.0 International License, which permits use, sharing, adaptation, distribution and reproduction in any medium or format, as long as you give appropriate credit to the original author(s) and the source, provide a link to the Creative Commons licence, and indicate if changes were made. The images or other third party material in this article are included in the article's Creative Commons licence, unless indicated otherwise in a credit line to the material. If material is not included in the article's Creative Commons licence and your intended use is not permitted by statutory regulation or exceeds the permitted use, you will need to obtain permission directly from the copyright holder. To view a copy of this licence, visit http://creativecommons.org/licenses/by/4.0/. 
Extraction procedure: acetone-M: $10 \mathrm{mM}$ ammonium format as extraction solvent

Spiked plasma samples were vortexed to ensure complete mixing of contents; $50 \mu$ l of internal standard $(1 \mu \mathrm{l} / \mathrm{ml}$ of bexarotene D4) solution was added into all respectively labeled empty RIA vials except blank. Five hundred microliters of plasma samples was added to the respective labeled RIA vials containing internal standard solution and vortexed. Two hundred microliters of buffer 1 was added to all the samples and vortexed. $2.5 \mathrm{ml}$ of extraction solvent was added to all the samples and capped. Samples were vibramaxed at $2000 \mathrm{rpm}$ for 10 min. Then, the samples were centrifuged at $3500 \mathrm{rpm}$ for $5 \mathrm{~min}$ in a refrigerated centrifuge between 2 and $8^{\circ} \mathrm{C}$. Two milliliters of supernatant was transferred into respective labeled RIA vials. All the samples were dried at $40{ }^{\circ} \mathrm{C}$ and 15 psi using LV evaporator. The dried residues get reconstituted with $300 \mu \mathrm{l}$ of mobile phase and vortexed. The phospholipid removal cartridges get conditioned with $1 \mathrm{ml}$ of acetone-M followed by $1 \mathrm{ml}$ of conditioning solution. The samples were loaded into cartridges and eluted into RIA vials. The samples were transferred into respective labeled auto injector vials and loaded into LC-MS/MS [3].

\section{Results}

\section{Method validation}

The method was validated according to $\mathrm{ICH}$ Guidelines Q2 (R1) with respect to system suitability, linearity, accuracy, precision, matrix effect, auto sampler carryover test, and recovery.

\section{System suitability}

Aqueous standard or extracted standard equivalent to middle level of CC standard concentration with internal standard was prepared. Six replicates from the same vial were injected into the chromatographic device. Mean, standard deviation, and percentage coefficient of variation for the retention time and area/area ratio were calculated.

\section{Linearity}

Different serial dilutions were repeated, and fresh aqueous standards (for CCs) were prepared. An appropriate regression model with minimal or no weighing $(1 / \mathrm{x}$ or $1 / \mathrm{x}^{2}$ ) was used. The standards were run in the LC-MS/ MS, and linearity was evaluated.

\section{Selectivity/specificity}

This is to check whether there is an interference in peak. Two sets of six normal lots of plasma and one hemolyzed were taken. The aqueous LLOQ dilution was prepared and was spiked in one set of six normal lots of plasma, one hemolyzed lot to achieve LLOQ concentration for analyte, and the specificity sample was processed. The internal standard dilution was prepared, and only $50 \mu \mathrm{L}$ of internal standard dilution was added to another set of six normal lots of plasma, one hemolyzed was processed for specificity samples. Selectivity samples were prepared in the presence of both analyte and internal standard using the six normal blank plasmas and one hemolyzed.

\section{Precision and accuracy}

The precision was determined by calculating percentage $\% \mathrm{CV}$ at each concentration level of QC sample, and the accuracy was determined by calculating the percentage of nominal value at each concentration level of QC samples.

\section{Ruggedness}

One P\&A batch was performed by employing the same instrument with different analysts and alternatively performed on different instruments of same make.

\section{Recovery}

The overall mean recovery, SD, and $\% \mathrm{CV}$ were calculated. The recovery experiment was carried out by inject the six replicates of unextracted low, medium, and high QC samples, along with freshly processed $\mathrm{CC}$ set and QCs (6 LOC, $6 \mathrm{MQC}$, and $6 \mathrm{HQ})$.

\section{Stability}

Evaluation of stability should be carried out to ensure that every step taken during sample preparation and sample analysis, as well as the storage conditions used, do not affect the concentration of the analyte. The stability tests conducted in method validation are as follows:

1. Stock solution stability: short-term stock solution stability, long-term stock solution stability

2. Stability in biological matrix: bench top stability, freeze-thaw stability, long-term stability, blood stability

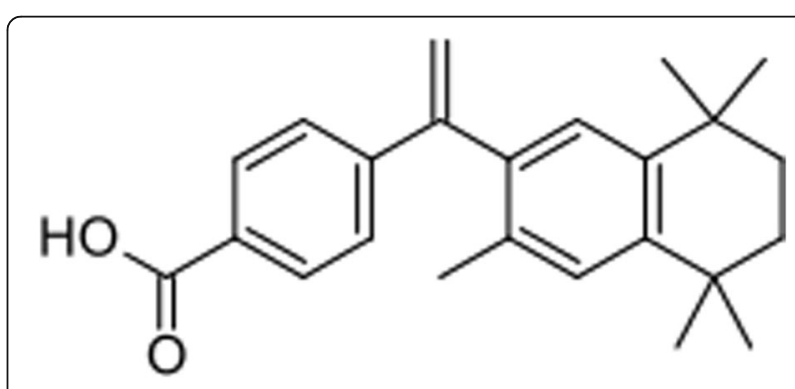

Fig. 1 Chemical structure of bexarotene. Formula: $\mathrm{C}_{24} \mathrm{H}_{28} \mathrm{O}_{2}$. Molar mass: $348.478 \mathrm{~g} / \mathrm{mol}$ 
Table 1 Specificity and selectivity for bexarotene and internal standard (MTR-BA-LC-MS/MS-05)

\begin{tabular}{|c|c|c|c|c|c|c|c|c|}
\hline \multirow[t]{2}{*}{ Plasma lot ID } & \multicolumn{2}{|c|}{ Specificity (blank) } & \multicolumn{2}{|c|}{ Selectivity (spiked LLOQ) } & \multicolumn{2}{|c|}{$\%$ interference in blank } & \multirow{2}{*}{$\begin{array}{l}\text { Area ratio } \\
\text { Analyte/IS }\end{array}$} & \multirow{2}{*}{$\begin{array}{l}\text { S/N ratio }(\geq 5) \\
\text { Analyte }\end{array}$} \\
\hline & Analyte & IS peak & Analyte & IS peak & Analyte $(<20 \%)$ & IS $(<5 \%)$ & & \\
\hline MAT-C-0293-III & 1805 & 2581 & 30,895 & $1,932,710$ & 5.8424 & 0.1335 & 0.0160 & 101.450 \\
\hline MAT-C-0577-III & 1274 & 930 & 31,179 & $1,861,940$ & 4.0861 & 0.0499 & 0.0167 & 118.834 \\
\hline MAT-C-0578-III & 1070 & 1798 & 29,447 & $1,823,809$ & 3.6336 & 0.0986 & 0.0161 & 75.245 \\
\hline MAT-C-0579-III & 513 & 1694 & 27,120 & $1,813,246$ & 1.8916 & 0.0934 & 0.0150 & 111.258 \\
\hline MAT-C-0580-III & 425 & 506 & 29,375 & $1,868,739$ & 1.4468 & 0.0271 & 0.0157 & 129.562 \\
\hline MAT-C-0586-III & 1528 & 1132 & 25,471 & $1,774,320$ & 5.9990 & 0.0638 & 0.0144 & 64.867 \\
\hline MAT-C-0544-I(H) & 1147 & 745 & 28,883 & $1,769,483$ & 3.9712 & 0.0421 & 0.0163 & 96.481 \\
\hline MAT-6188-I(L) & 1396 & 2263 & 28,664 & $1,815,377$ & 4.8702 & 0.1247 & 0.0158 & 95.259 \\
\hline \multirow[t]{4}{*}{ MAT-6198-IX (heparin) } & 453 & 1361 & 27,446 & $1,825,500$ & 1.6505 & 0.0746 & 0.0150 & 81.708 \\
\hline & & & & Mean & 3.71016 & 0.07863 & 0.01567 & \\
\hline & & & & & & SD & 0.000731 & \\
\hline & & & & & & $\% \mathrm{CV}$ & 4.67 & \\
\hline
\end{tabular}

\section{Discussion}

\section{Method development}

The LCMS/MS procedure was optimized for the estimation of bexarotene with the mobile phase of acetonitrile: buffer 1 (90:10, v/v); the optimum flowrate was $1 \mathrm{ml} / \mathrm{min}$ with a column oven temperature and autosampler temperature of $40^{\circ} \mathrm{C}$ and $10^{\circ} \mathrm{C}$ respectively. Retention time of analyte is $2.75 \pm 0.3 \mathrm{~min}$, and IS is $2.73 \pm 0.3 \mathrm{~min}$.

\section{Specificity and selectivity}

Selectivity was evaluated by analyzing a total of nine lots on the instrument [17] obtained from independent sources (Table 1). No significant interferences were observed at the retention times of analyte and internal standard (see Table 3).

\section{Signal-to-noise $(\mathrm{S} / \mathrm{N})$ ratio}

The signal-to-noise ratio was determined for bexarotene at LLOQ concentrations in nine independent lots of $\mathrm{K}_{2}$ EDTA human normal plasma including one lot of hemolyzed plasma, one lot of heparin plasma, and one lot of lipemic plasma [8] demonstrating acceptable $\mathrm{S} / \mathrm{N}$ intensity.

\section{Carryover test}

Carryover is calculated as the percentage peak area observed in a processed blank plasma injected immediately after a processed ULOQ calibration standard, which was used from PA-01 batch sample analysis. No significant carryover was observed for bexarotene and internal standard (see in Table 2).

\section{Matrix effect and matrix factor}

Matrix factor and matrix effect were calculated, and results are given in Tables 3 and 4.

\section{Linearity}

Linearity established [9] by preparing an eight-point standard calibration curve in $\mathrm{K}_{2}$ EDTA human plasma covering the bexarotene concentration ranges from 1.0440 to $351.9320 \mu \mathrm{g} / \mathrm{mL}$ using bexarotene D4 as internal standard. The calibration curve was shown to be linear for bexarotene as shown in Fig. 2; the results are seen in Table 5.

\section{Weighting scheme}

The absolute values of residuals of the back-calculated bexarotene calibration standards for the curve were tabulated, and the sum of the absolute values of the residuals was calculated for each weighting factor. The weighting factor of $1 / \mathrm{X}^{2}$ provided the least sum value

Table 2 Carry over test

\begin{tabular}{lll}
\hline Sample ID & Analyte peak area & IS peak area \\
\hline Extracted blank & 0 & 645 \\
Extracted LLOQ+IS & 6166 & 456,817 \\
Extracted ULOQ+IS & $1,955,374$ & 413,370 \\
Extracted blank I & 0 & 504 \\
Extracted blank II & 0 & 419 \\
Average of extracted blank & 0 & 462 \\
\% carry over & $\mathbf{0 . 0 0}$ & $\mathbf{- 0 . 0 4}$ \\
\hline
\end{tabular}


Table 3 Matrix effect and matrix factor for bexarotene at LQC level

\begin{tabular}{|c|c|c|c|c|c|c|c|c|c|}
\hline \multirow[t]{2}{*}{ Plasma lot ID } & \multicolumn{2}{|c|}{ Aqueous sample } & \multicolumn{2}{|c|}{ Spiked sample } & \multirow{2}{*}{$\begin{array}{l}\text { Matrix } \\
\text { factor } \\
\text { of } \\
\text { analyte }\end{array}$} & \multirow{2}{*}{$\begin{array}{l}\text { Matrix } \\
\text { factor } \\
\text { of IS }\end{array}$} & \multirow{2}{*}{$\begin{array}{l}\text { IS } \\
\text { normalized } \\
\text { matrix } \\
\text { factor }\end{array}$} & \multicolumn{2}{|l|}{ Area ratio } \\
\hline & $\begin{array}{l}\text { Analyte } \\
\text { area }\end{array}$ & IS area & $\begin{array}{l}\text { Analyte } \\
\text { area }\end{array}$ & IS area & & & & $\begin{array}{l}\text { Aqueous } \\
\text { sample }\end{array}$ & $\begin{array}{l}\text { Spiked } \\
\text { sample }\end{array}$ \\
\hline MAT6223-I & 48,348 & $1,336,922$ & 45,987 & $1,317,311$ & 1.08 & 1.03 & 1.05 & 0.0362 & 0.0349 \\
\hline MAT6224-I & 42,001 & $1,271,968$ & 42,437 & $1,277,296$ & 0.99 & 1.00 & 0.99 & 0.033 & 0.0332 \\
\hline MAT6225-I & 43,318 & $1,289,768$ & 40,402 & $1,257,917$ & 0.95 & 0.98 & 0.97 & 0.0336 & 0.0321 \\
\hline MAT6220-I & 37,838 & $1,240,867$ & 42,187 & $1,239,881$ & 0.99 & 0.97 & 1.02 & 0.0305 & 0.0340 \\
\hline MAT6204-I & 41,861 & $1,246,249$ & 43,795 & $1,222,111$ & 1.03 & 0.96 & 1.07 & 0.0336 & 0.0358 \\
\hline MAT6205-I & 42,559 & $1,278,563$ & 41,373 & $1,221,142$ & 0.97 & 0.96 & 1.01 & 0.0333 & 0.0339 \\
\hline MATC-0544-XII(H) & & & 43,332 & $1,289,828$ & 1.02 & 1.01 & 1.01 & & 0.0336 \\
\hline MAT6188-IX (L) & & & 42,395 & $1,282,621$ & 0.99 & 1.00 & 0.99 & & 0.0331 \\
\hline $\begin{array}{l}\text { MAT6198-(X)-Heparin } \\
\text { Plasma }\end{array}$ & & & 43,054 & $1,277,420$ & 1.01 & 1.00 & 1.01 & & 0.0337 \\
\hline Mean & $42,654.16$ & $1,277,389.5$ & & & 1.003 & 0.990 & 1.01333 & 0.033 & 0.033 \\
\hline SD & & & & & 0.037 & 0.023 & 0.03082 & & \\
\hline$\% \mathrm{CV}$ & & & & & 3.76 & 2.42 & 3.04 & & \\
\hline
\end{tabular}

with residuals of calibration curve standards. Hence, 1/ $\mathrm{X}^{2}$ was selected to use for this validation. See the results in Table 6 .

\section{Sensitivity}

The sensitivity for bexarotene at LLOQ level in $\mathrm{K}_{2}$ EDTA human plasma determined based on the analysis of six replicates of LLOQ $(1.0440 \mathrm{ng} / \mathrm{mL})$ samples was prepared and analyzed against calibration curve standards. See the results in Table 7.

\section{Intra-batch precision and accuracy of bexarotene} See the results in Table 8 .

\section{Ruggedness}

Accuracy, assay precision, and accuracy value for ruggedness batch (PA-03) were determined by

Table 4 Matrix effect and matrix factor for bexarotene at LQC level (MTR-BA-LC-MS/MS-05)

\begin{tabular}{|c|c|c|c|c|c|c|c|c|c|}
\hline & \multicolumn{2}{|c|}{ Aqueous sample } & \multicolumn{2}{|c|}{ Spiked sample } & \multirow{2}{*}{$\begin{array}{l}\text { Matrix } \\
\text { factor } \\
\text { of } \\
\text { analyte }\end{array}$} & \multirow{2}{*}{$\begin{array}{l}\text { Matrix } \\
\text { factor } \\
\text { of IS }\end{array}$} & \multirow{2}{*}{$\begin{array}{l}\text { IS } \\
\text { normalized } \\
\text { matrix } \\
\text { factor }\end{array}$} & \multicolumn{2}{|l|}{ Area ratio } \\
\hline & $\begin{array}{l}\text { Analyte } \\
\text { area }\end{array}$ & IS area & $\begin{array}{l}\text { Analyte } \\
\text { area }\end{array}$ & IS area & & & & $\begin{array}{l}\text { Aqueous } \\
\text { sample }\end{array}$ & $\begin{array}{l}\text { Spiked } \\
\text { sample }\end{array}$ \\
\hline MAT-6223-I & 48,348 & $1,336,922$ & 45,987 & $1,317,311$ & 1.08 & 1.03 & 1.05 & 0.036 & 0.03 \\
\hline MAT-6224-I & 42,001 & $1,271,968$ & 42,437 & $1,277,296$ & 0.99 & 1.00 & 0.99 & 0.033 & 0.03 \\
\hline MAT-6225-I & 43,318 & $1,289,768$ & 40,402 & $1,257,917$ & 0.95 & 0.98 & 0.97 & 0.033 & 0.03 \\
\hline MAT-6220-I & 37,838 & $1,240,867$ & 42,187 & $1,239,881$ & 0.99 & 0.97 & 1.02 & 0.030 & 0.03 \\
\hline MAT-6204-I & 41,861 & $1,246,249$ & 43,795 & $1,222,111$ & 1.03 & 0.96 & 1.07 & 0.033 & 0.03 \\
\hline MAT-6205-I & 42,559 & $1,278,563$ & 41,373 & $1,221,142$ & 0.97 & 0.96 & 1.01 & 0.033 & 0.03 \\
\hline MAT-C-0544-XII(H) & & & 43,332 & $1,289,828$ & 1.02 & 1.01 & 1.01 & & 0.03 \\
\hline MAT-6188-IX (L) & & & 42,395 & $1,282,621$ & 0.99 & 1.00 & 0.99 & & 0.03 \\
\hline $\begin{array}{l}\text { MAT-6198-(X)-heparin } \\
\text { plasma }\end{array}$ & & & 43,054 & $1,277,420$ & 1.01 & 1.00 & 1.01 & & 0.03 \\
\hline Mean & $42,654.16$ & $1,277,389$ & & & 1.003 & 0.99 & 1.01333 & 0.033 & 0.03 \\
\hline SD & & & & & 0.037 & 0.023 & 0.030822 & & \\
\hline$\% C V$ & & & & & 3.76 & 2.42 & 3.04 & & \\
\hline
\end{tabular}




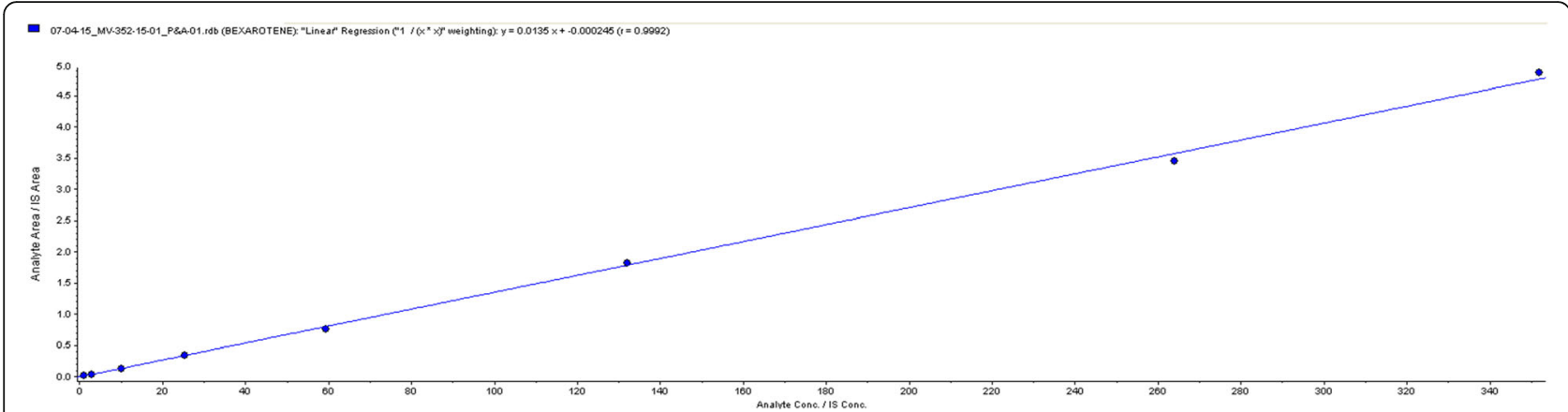

Fig. 2 Calibration curve

Table 5 Back-calculated concentrations of bexarotene for calibration curve standards

\begin{tabular}{|c|c|c|c|c|c|c|c|c|c|c|c|}
\hline Standard ID & $A$ & B & $\mathrm{C}$ & $\mathrm{D}$ & $E$ & $F$ & G & $\mathrm{H}$ & Slope & Intercept & $r^{2}$ \\
\hline Actual concentration $(\mathrm{ng} / \mathrm{mL})$ & 1.044 & 2.7760 & 10.096 & 25.240 & 59.390 & 131.97 & 263.950 & 351.93 & & & \\
\hline PA-01 & 1.019 & 2.9590 & 9.9554 & 25.592 & 56.512 & 134.04 & 255.361 & 359.85 & 0.013 & -0.0002 & 0.998 \\
\hline PA-02 & 1.052 & 2.7224 & 9.9902 & 25.479 & 57.677 & 132.92 & 272.270 & 352.68 & 0.013 & 0.0011 & 0.999 \\
\hline Mean & 1.0362 & 2.8407 & 9.9728 & 25.535 & 57.095 & 133.48 & 263.81 & 356.27 & & & \\
\hline SD & 0.02319 & 0.167301 & 0.024607 & 0.079974 & 0.823921 & 0.787929 & 11.956327 & 5.072289 & & & \\
\hline$\% C V$ & 2.24 & 5.89 & 0.25 & 0.31 & 1.44 & 0.59 & 4.53 & 1.42 & & & \\
\hline \%Nominal & 99.25 & 102.33 & 98.78 & 101.17 & 96.14 & 101.14 & 99.95 & 101.23 & & & \\
\hline
\end{tabular}

Table 6 Weighting scheme

\begin{tabular}{ll}
\hline Weighting-1/ $\mathbf{X}^{\mathbf{2}}$ & Weighting $\mathbf{- 1 / \mathbf { X }}$ \\
Absolute values of residuals & Absolute values of residuals \\
\hline 1.83 & 3.01 \\
5.23 & 4.99 \\
0.10 & 0.03 \\
1.67 & 1.45 \\
4.85 & 4.60 \\
4.66 & 4.94 \\
0.17 & 0.44 \\
1.61 & 1.34 \\
$\mathbf{2 0 . 1 2}$ & $\mathbf{2 0 . 8 0}$ \\
\hline
\end{tabular}

Table 7 Sensitivity

\begin{tabular}{ll}
\hline Parameters & LLOQ \\
\hline Actual concentration $(\mathbf{n g} / \mathbf{m L})$ & $\mathbf{1 . 0 4 4 0}$ \\
PA-01 (MTR-BA-LC-MS/MS-19) & 1.0198 \\
PA-02 (MTR-BA-LC-MS/MS-19) & 1.0526 \\
PA-03 (MTR-BA-LC-MS/MS-05) & 1.0710 \\
Mean & $\mathbf{1 . 0 4 7 8 0}$ \\
SD & $\mathbf{0 . 0 2 5 9 3 5}$ \\
\hline
\end{tabular}


Table $\mathbf{8}$ Intra-batch precision and accuracy of bexarotene

\begin{tabular}{|c|c|c|c|c|c|}
\hline QC ID & LOQQC & LQC & INTQC & MQC & HQC \\
\hline Actual concentration $(\mathrm{ng} / \mathrm{mL})$ & 1.0500 & 2.7800 & 25.2700 & 131.6160 & 263.2320 \\
\hline \multirow{6}{*}{$\begin{array}{l}\text { Calculated concentration (ng/mL) (MTR-BA-LC-MS/MS-19) PA-01 } \\
\text { (06 Apr 2015) }\end{array}$} & 1.1639 & 2.9471 & 25.8272 & 135.3234 & 277.1292 \\
\hline & 1.1622 & 2.8307 & 26.0325 & 128.5281 & 276.5622 \\
\hline & 1.0975 & 2.9013 & 25.3157 & 131.3295 & 272.8455 \\
\hline & 1.0866 & 2.8823 & 25.3351 & 132.7338 & 267.9314 \\
\hline & 1.1758 & 3.0348 & 26.1218 & 136.8898 & 264.0381 \\
\hline & 1.1043 & 2.9858 & 26.5157 & 133.2429 & 277.9904 \\
\hline Mean & 1.13172 & 2.93033 & 25.85800 & 133.00792 & 272.74947 \\
\hline SD & 0.039664 & 0.073957 & 0.469270 & 2.947768 & 5.662107 \\
\hline$\% \mathrm{CV}$ & 3.50 & 2.52 & 1.81 & 2.22 & 2.08 \\
\hline \%nominal & 107.78 & 105.41 & 102.33 & 101.06 & 103.62 \\
\hline \multirow{6}{*}{$\begin{array}{l}\text { Calculated concentration (ng/mL) (MTR-BA-LC-MS/MS-19) PA-02 } \\
\text { (07 Apr 2015) }\end{array}$} & 1.0709 & 2.7864 & 26.1883 & 134.5485 & 270.4602 \\
\hline & 1.0005 & 2.8122 & 25.8356 & 134.5475 & 274.3989 \\
\hline & 0.8813 & 2.8064 & 25.3888 & 138.3150 & 266.9063 \\
\hline & 1.0456 & 2.8642 & 25.3942 & 133.0021 & 272.3938 \\
\hline & 1.1056 & 2.9071 & 25.5199 & 132.3109 & 266.4461 \\
\hline & 1.0615 & 2.8626 & 25.6117 & 136.5209 & 273.7517 \\
\hline Mean & 1.02757 & 2.83982 & 25.65642 & 134.87415 & 270.72617 \\
\hline SD & 0.079461 & 0.045549 & 0.308551 & 2.228847 & 3.417745 \\
\hline$\% \mathrm{CV}$ & 7.73 & 1.60 & 1.20 & 1.65 & 1.26 \\
\hline \%Nominal & 97.86 & 102.15 & 101.53 & 102.48 & 102.85 \\
\hline
\end{tabular}

Table 9 Ruggedness (MTR-BA-LC-MS/MS-05)

\begin{tabular}{lllllllll}
\hline Standard & A & B & C & D & E & F & G & H \\
\hline Actual conc $(\mathrm{ng} / \mathrm{mL})$ & 1.0440 & 2.7760 & 10.0960 & 25.2400 & 59.3900 & 131.9760 & 263.9500 & 351.9320 \\
Calculated conc $(\mathrm{ng} / \mathrm{mL})$ & 1.0710 & 2.5793 & 10.0606 & 25.4973 & 62.1811 & 131.0732 & 264.5648 & 350.4726 \\
\%nominal & 102.59 & 92.91 & 99.65 & 101.02 & 104.70 & 99.32 & 100.23 & 99.59 \\
\hline
\end{tabular}

Table 10 Precision

\begin{tabular}{llllll}
\hline QC ID & LOQQC & LQC & INTQC & MQC & HQC \\
\hline Actual concentration $(\mathrm{ng} / \mathrm{mL})$ & $\mathbf{1 . 0 5 0 0}$ & $\mathbf{2 . 7 8 0 0}$ & $\mathbf{2 5 . 2 7 0 0}$ & $\mathbf{1 3 1 . 6 1 6 0}$ & $\mathbf{2 6 3 . 2 3 2 0}$ \\
Calculated concentration $(\mathrm{ng} / \mathrm{mL})$ & 1.2696 & 2.6583 & 28.1908 & 140.8541 & 270.9386 \\
& 0.9477 & 2.6411 & 26.3654 & 142.1994 & 276.2971 \\
& 0.9881 & 2.6797 & 27.1780 & 140.3503 & 277.4336 \\
& 1.0373 & 2.7942 & 27.6385 & 138.9731 & 275.7356 \\
& 1.0366 & 2.6154 & 27.3704 & 141.6173 & 269.9140 \\
Mean & 1.0515 & 2.8529 & 27.9700 & 141.5019 & $\mathbf{1 4 0 . 9 1 6 0 2}$ \\
SD & $\mathbf{1 . 0 5 5 1 3}$ & $\mathbf{2 . 7 0 6 9 3}$ & $\mathbf{2 7 . 4 5 2 1 8}$ & $\mathbf{2 7 3 . 2 9 3 2 8}$ \\
CV & $\mathbf{0 . 1 1 1 9 8 8}$ & $\mathbf{0 . 0 9 4 5 9 5}$ & $\mathbf{0 . 6 4 9 8 2 5}$ & $\mathbf{1 . 1 4 7 2 5 1}$ & $\mathbf{3 . 5 7 5 9 1 3}$ \\
Nominal & $\mathbf{1 0 . 6 1}$ & $\mathbf{3 . 4 9}$ & $\mathbf{2 . 3 7}$ & $\mathbf{0 . 8 1}$ & $\mathbf{1 . 3 1}$ \\
\hline
\end{tabular}


analyzing six replicates each of LOQQC, LQC, INTQ $\mathrm{C}, \mathrm{MQC}$, and HQC samples using different instrument (MTR-BA-LC-MS/MS-05) of the same make and model (UPLC with Triple Quad API 4000), different analytical column (BAC-0644), and different analyst (Table 9).

Intercept $=0.0019$, Slope $=0.0118, r^{2}=0.9996$

\section{Precision}

The precision of the assay was measured by the percentage co-efficient of variation over the concentration range of LOQQC, LQC, INTQC, MQC, and HQC samples of bexarotene during the course of partial validation. See the results in Table 10.

Table 11 Recovery of bexarotene

\begin{tabular}{|c|c|c|}
\hline Quality control sample ID & Aqueous analyte area & Extracted analyte area \\
\hline \multirow[t]{6}{*}{ LQC } & 16,201 & 17,052 \\
\hline & 16,045 & 16,462 \\
\hline & 20,592 & 16,952 \\
\hline & 20,215 & 16,941 \\
\hline & 20,098 & 20,900 \\
\hline & 19,423 & 17,689 \\
\hline Mean & 18,762 & 17,666 \\
\hline$\%$ recovery & 94.16 & \\
\hline \multirow[t]{6}{*}{ MQC } & 766,900 & 764,709 \\
\hline & 766,878 & 748,447 \\
\hline & 756,525 & 743,886 \\
\hline & 769,926 & 744,175 \\
\hline & 766,882 & 741,445 \\
\hline & 757,276 & 753,506 \\
\hline Mean & 764,065 & 749,361 \\
\hline$\%$ recovery & 98.08 & \\
\hline \multirow[t]{6}{*}{ HQC } & $1,548,386$ & $1,398,325$ \\
\hline & $1,543,021$ & $1,373,844$ \\
\hline & $1,562,977$ & $1,421,433$ \\
\hline & $1,573,624$ & $1,379,191$ \\
\hline & $1,557,258$ & $1,372,197$ \\
\hline & $1,542,212$ & $1,352,389$ \\
\hline Mean & $1,554,580$ & $1,382,897$ \\
\hline$\%$ recovery & 88.96 & \\
\hline \multicolumn{3}{|l|}{ Recovery result } \\
\hline LQC & 94.16 & \\
\hline MQC & 98.08 & \\
\hline HQC & 88.96 & \\
\hline Mean & 93.73 & \\
\hline SD & 4.57 & \\
\hline$\% \mathrm{CV}$ & 4.88 & \\
\hline
\end{tabular}

\section{Recovery of bexarotene and IS}

The recovery of bexarotene was determined by comparing the detector response of analyte at three distinct levels of extracted low-, medium-, and high-quality control samples of PA-01 with detector response obtained from unextracted aqueous quality control samples at low, medium, and high level respectively. See the results in Tables 11 and 12.

IS recovery $=95.72 \%$

\section{Stability}

\section{Freeze-thaw stability}

Six replicates of bexarotene samples at LQC and HQC concentration in $\mathrm{K}_{2}$ EDTA human plasma were analyzed after four freeze-thaw (FT4) cycles. See the results in Table 13.

\section{Bench top stability}

Bench top stability of bexarotene in $\mathrm{K}_{2}$ EDTA human plasma was evaluated at room temperature. Six replicates of LQC and HQC samples were processed after keeping the samples on bench for about $12.30 \mathrm{~h}$. See the results in Table 14.

Table 12 Recovery of internal standard

\begin{tabular}{lll}
\hline $\begin{array}{l}\text { Quality control } \\
\text { Sample ID }\end{array}$ & Aqueous IS area & Extracted IS area \\
\hline LQC & 412,999 & 430,053 \\
& 412,341 & 432,371 \\
& 406,330 & 434,330 \\
& 409,620 & 436,925 \\
& 412,767 & 511,773 \\
& 405,852 & 440,319 \\
MQC & 463,632 & 417,498 \\
& 456,101 & 430,227 \\
& 465,696 & 418,482 \\
& 465,204 & 414,215 \\
& 465,835 & 400,164 \\
HQC & 462,232 & 417,807 \\
& 421,616 & 372,759 \\
& 423,021 & 366,983 \\
& 416,624 & 384,868 \\
& 413,104 & 380,280 \\
& 427,069 & 383,931 \\
& 424,578 & 359,396 \\
& $\mathbf{4 3 1 , 3 6 7 . 8 3 3 3 3}$ & $\mathbf{4 1 2 , 9 1 0 . 0 5 5 6 0}$ \\
\hline
\end{tabular}


Table 13 Freeze-thaw stability for bexarotene (at $-70^{\circ} \mathrm{C} \pm 15^{\circ} \mathrm{C}$ and $-30^{\circ} \mathrm{C} \pm 10$ )

\begin{tabular}{|c|c|c|c|c|}
\hline \multirow[t]{2}{*}{$\overline{Q C ~ I D ~}$} & \multicolumn{2}{|c|}{$-70^{\circ} \mathrm{C} \pm 15^{\circ} \mathrm{C}$} & \multicolumn{2}{|c|}{$-30^{\circ} \mathrm{C} \pm 10^{\circ} \mathrm{C}$} \\
\hline & LQC FT4 & HQC FT4 & LQC FT4 & HQC FT4 \\
\hline Actual concentration $(\mathrm{ng} / \mathrm{mL})$ & 2.7800 & 263.2320 & 2.7800 & 263.2320 \\
\hline \multirow[t]{6}{*}{ Calculated concentrations ( $\mathrm{ng} / \mathrm{mL})$} & 2.7739 & 277.0834 & 2.9870 & 270.1236 \\
\hline & 2.7193 & 264.5538 & 2.8392 & 272.0022 \\
\hline & 2.9684 & 272.1494 & 2.6626 & 263.9169 \\
\hline & 2.6266 & 269.6594 & 2.7717 & 276.9206 \\
\hline & 2.9136 & 268.7336 & 2.9150 & 266.3756 \\
\hline & 2.8143 & 271.7938 & 2.7318 & 262.5824 \\
\hline Mean & 2.80268 & 270.66223 & 2.81788 & 268.65355 \\
\hline SD & 0.125448 & 4.165615 & 0.120119 & 5.407447 \\
\hline$\% \mathrm{CV}$ & 4.48 & 1.54 & 4.26 & 2.01 \\
\hline \%nominal & 100.82 & 102.82 & 101.36 & 102.06 \\
\hline
\end{tabular}

\section{Auto sampler stability for bexarotene}

Six replicates of LQC and HQC samples were processed and kept stored in auto sampler at $10{ }^{\circ} \mathrm{C}$ for $96.80 \mathrm{~h} \mathrm{[11]}$. See the results in Tables 15 and 16.

\section{Long-term stock solution stability for bexarotene}

Stock solution bexarotene with concentration of $975.6417 \mu \mathrm{g} / \mathrm{mL}$ was kept in the refrigerator for 14 days [12]. A fresh stock of $986.1296 \mu \mathrm{g} / \mathrm{mL}$ was prepared on the day of analysis. Both stocks were diluted to LQC and HQC equivalent concentration of $0.1317 \mu \mathrm{g} / \mathrm{mL}$ and $0.1331 \mu \mathrm{g} / \mathrm{mL}$ and 13.1712 and $13.3127 \mu \mathrm{g} / \mathrm{mL}$ for stored and fresh stock respectively. The area ratios of stability stock solution at LQC and HQC level were compared against freshly prepared stock solution LQC and HQC level. See the results in Tables 17 and 18.

\section{Limit of detection}

From LLOQ sample $(1.0540 \mathrm{ng} / \mathrm{mL})$, four different lower concentrations $(0.8440,0.6340,0.4240$, and $0.2120 \mathrm{ng} / \mathrm{mL}$ ) including five times the lower concentration (LOD dilution) were prepared, and six replicates of these samples were analyzed. So the selected LLOQ (approx. $1.0540 \mathrm{ng} / \mathrm{mL}$ ) was more suitable to quantify bexarotene in plasma using LC-MS/MS.

Reinjection reproducibility [14-16]

CC standards, LQC, and HQC samples of PA-02 were reinjected after $08.95 \mathrm{~h}$. Percentage nominal for LQC
Table 14 Bench top stability for bexarotene

\begin{tabular}{lll}
\hline Stability hours & $\mathbf{1 2 . 3 0 ~} \mathbf{~}$ & \\
\cline { 2 - 3 } QC ID & LQC (stability) & HQC (stability) \\
\hline Actual concentration $(\mathbf{n g} / \mathrm{mL})$ & $\mathbf{2 . 7 8 0 0}$ & $\mathbf{2 6 3 . 2 3 2 0}$ \\
Calculated concentration $(\mathrm{ng} / \mathrm{mL})$ & 2.7480 & 272.6737 \\
& 2.7554 & 268.0237 \\
& 2.8146 & 268.2582 \\
& 2.8589 & 258.8974 \\
& 2.8595 & 263.2458 \\
& 2.7803 & 266.8280 \\
Mean & $\mathbf{2 . 8 0 2 7 8}$ & $\mathbf{2 6 6 . 3 2 1 1 3}$ \\
SD & $\mathbf{0 . 0 4 9 5 1 2}$ & $\mathbf{4 . 7 2 9 3 2 3}$ \\
\%CV & $\mathbf{1 . 7 7}$ & $\mathbf{1 . 7 8}$ \\
\%nominal & $\mathbf{1 0 0 . 8 2}$ & $\mathbf{1 0 1 . 1 7}$ \\
\hline
\end{tabular}

Table 15 Auto sampler stability for bexarotene

\begin{tabular}{lll}
\hline Stability hours & $\mathbf{9 6 . 8 0} \mathrm{h}$ \\
\cline { 2 - 3 } QC ID & LQC (stability) & HQC (stability) \\
\hline Actual concentration $(\mathrm{ng} / \mathrm{mL})$ & 2.7800 & 263.2320 \\
Calculated concentration $(\mathrm{ng} / \mathrm{mL})$ & 2.8654 & 274.3981 \\
& 2.8602 & 273.6167 \\
& 2.8930 & 272.6483 \\
& 2.9033 & 266.1408 \\
& 2.7949 & 270.6783 \\
& 2.7693 & 267.6223 \\
Mean & $\mathbf{2 . 8 4 7 6 8}$ & $\mathbf{2 7 0 . 8 5 0 7 5}$ \\
SD & $\mathbf{0 . 0 5 3 9 4 0}$ & $\mathbf{3 . 3 4 9 6 7 5}$ \\
\%CV & $\mathbf{1 . 8 9}$ & $\mathbf{1 . 2 4}$ \\
\%nominal & $\mathbf{1 0 2 . 4 3}$ & $\mathbf{1 0 2 . 8 9}$ \\
\hline
\end{tabular}


Table 16 Auto sampler stability for internal standard

\begin{tabular}{lll}
\hline Stability hours & $\mathbf{0} \mathbf{h}$ & $\mathbf{9 6 . 8 0} \mathbf{h}$ \\
QC ID & CS (IS area) & Stability samples (IS area) \\
\hline LQC & 314,806 & 326,389 \\
& 371,920 & 288,982 \\
& 313,781 & 303,778 \\
& 307,364 & 301,846 \\
& 368,512 & 314,200 \\
& 289,032 & 327,489 \\
HQC & 296,546 & 311,897 \\
& 293,776 & 307,720 \\
& 363,070 & 288,166 \\
& 320,306 & 313,358 \\
& 315,974 & 351,347 \\
Mean & 335,867 & 297,954 \\
\hline stability & $\mathbf{3 2 4 , 2 4 6 . 1 6 6 6 7}$ & $\mathbf{3 1 1 , 0 9 3 . 8 3 3 3 3}$ \\
\hline
\end{tabular}

and HQC for bexarotene was 98.99 and $100.30 \%$, respectively. The percentage CV for LQC and HQC for bexarotene was 3.66 and $1.97 \%$, respectively. See the results in Table 19.

\section{Conclusion}

Bioanalytical method is developed and validated as per ICH guidelines for the estimation of bexarotene in human plasma by using LC-MS/MS. The mobile phase was selected after trying various combinations of polar solvents. The proportion of solvents and variation of buffers were found to be quite critical as slight variation in it adversely affected the resolution of peaks. Considering all the facts, the validation parameter was finally fixed for this method. The

Table 17 Long-term stock solution stability for analyte LQC

\begin{tabular}{|c|c|c|c|c|c|c|}
\hline \multirow{2}{*}{$\begin{array}{l}\text { S. } \\
\text { No. }\end{array}$} & \multicolumn{3}{|c|}{ Solution 1 (14 days) } & \multicolumn{3}{|c|}{ Solution 3 ( 0 day) } \\
\hline & $\begin{array}{l}\text { Analyte } \\
\text { area }\end{array}$ & IS area & $\begin{array}{l}\text { Area } \\
\text { ratio }\end{array}$ & $\begin{array}{l}\text { Analyte } \\
\text { area }\end{array}$ & IS area & $\begin{array}{l}\text { Area } \\
\text { ratio }\end{array}$ \\
\hline 1 & 47,688 & $1,336,789$ & 0.0357 & 41,817 & $1,175,778$ & 0.0356 \\
\hline 2 & 48,589 & $1,346,976$ & 0.0361 & 41,215 & $1,151,561$ & 0.0358 \\
\hline 3 & 48,301 & $1,361,402$ & 0.0355 & 41,798 & $1,116,568$ & 0.0374 \\
\hline 4 & 45,066 & $1,366,924$ & 0.0330 & 41,422 & $1,148,997$ & 0.0361 \\
\hline 5 & 47,950 & $1,385,003$ & 0.0346 & 39,561 & $1,164,781$ & 0.0340 \\
\hline \multirow[t]{2}{*}{6} & 48,638 & $1,388,752$ & 0.0350 & 41,488 & $1,142,573$ & 0.0363 \\
\hline & & Mean & 0.03498 & & Mean & 0.03587 \\
\hline
\end{tabular}

Table 18 Long-term stock solution stability for internal standard LQC

\begin{tabular}{|c|c|c|c|c|c|c|}
\hline \multirow{2}{*}{$\begin{array}{l}\text { S. } \\
\text { No. }\end{array}$} & \multicolumn{3}{|c|}{ Solution 2 (14 days) } & \multicolumn{3}{|c|}{ Solution 3 (0 day) } \\
\hline & Analyte area & $\begin{array}{l}\text { IS } \\
\text { area }\end{array}$ & $\begin{array}{l}\text { Area } \\
\text { ratio }\end{array}$ & $\begin{array}{l}\text { Analyte } \\
\text { area }\end{array}$ & $\begin{array}{l}\text { IS } \\
\text { area }\end{array}$ & $\begin{array}{l}\text { Area } \\
\text { ratio }\end{array}$ \\
\hline 1 & 58,926 & $1,650,164$ & 28.00401 & 41,817 & $1,175,778$ & 28.11723 \\
\hline 2 & 58,933 & $1,642,596$ & 27.87226 & 41,215 & $1,151,561$ & 27.94034 \\
\hline 3 & 58,486 & $1,695,246$ & 28.98550 & 41,798 & $1,116,568$ & 26.71343 \\
\hline 4 & 55,830 & $1,678,648$ & 30.06713 & 41,422 & $1,148,997$ & 27.73881 \\
\hline 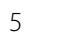 & 59,683 & $1,697,852$ & 28.44783 & 39,561 & $1,164,781$ & 29.44266 \\
\hline & 62,675 & $1,732,273$ & 27.63898 & 41,488 & $1,142,573$ & 27.53984 \\
\hline & & Mean & 28.50262 & & Mean & 27.91538 \\
\hline
\end{tabular}

bioanalytical method, a selective and sensitive liquid chromatography-mass spectrometry method to quantitate bexarotene in $\mathrm{K}_{2} \mathrm{EDTA}$ human plasma over the concentration range from 1.0440 to $351.9320 \mathrm{ng} / \mathrm{mL}$, was successfully validated. This method is suitable for sample analysis to support bioequivalence/bioavailability and/or pharmacokinetic studies involving formulations of bexarotene.

\section{Abbreviations \\ FDA: US Food and Drug Administration; RXR: The retinoid X receptor; EMA: European Medicines Agency; CTCL: Cutaneous T cell lymphoma; RPLC: Reverse phase liquid chromatography; HILIC: Hydrophilic interaction liquid chromatography; LC-MS: Liquid chromatography and mass spectroscopy; RARs: Retinoic acid receptors; IS: Internal standard; HPLC: High performance liquid chromatography; RIA: Radioimmunoassay; ICH: International Council for Harmonisation; LLOQ: Lower limit of quantification; ULOQ: Upper limit of quantification}

Table 19 Reinjection reproducibility for bexarotene

\begin{tabular}{lllll}
\hline Batch ID & \multicolumn{3}{l}{ Reinjection reproducibility } \\
\cline { 2 - 3 } & \multicolumn{3}{l}{ PA-02 samples } & \multicolumn{2}{l}{$\begin{array}{l}\text { Reinjected samples } \\
\text { (08.95 } \mathbf{h})\end{array}$} \\
\cline { 2 - 3 } \cline { 5 - 6 } & LQC & HQC & LQC & HQC \\
\cline { 1 - 2 } $\begin{array}{l}\text { Actual concentrations } \\
\text { (ng/mL) }\end{array}$ & $\mathbf{2 . 7 8 0 0}$ & $\mathbf{2 6 3 . 2 3 2 0}$ & $\mathbf{2 . 7 8 0 0}$ & $\mathbf{2 6 3 . 2 3 2 0}$ \\
Calculated & & & & \\
concentration $(\mathrm{ng} / \mathrm{mL})$ & 2.7864 & 270.4602 & 2.6865 & 266.3424 \\
& 2.8122 & 274.3989 & 2.8310 & 269.9017 \\
& 2.8064 & 266.9063 & 2.8128 & 260.9096 \\
& 2.8642 & 272.3938 & 2.7216 & 263.9505 \\
& 2.9071 & 266.4461 & 2.8619 & 267.5449 \\
Mean & 2.8626 & 273.7517 & 2.5983 & 255.4501 \\
SD & $\mathbf{2 . 8 3 9 8 2}$ & $\mathbf{2 7 0 . 7 2 6 1 7}$ & $\mathbf{2 . 7 5 2 0 2}$ & $\mathbf{2 6 4 . 0 1 6 5 3}$ \\
\%CV & $\mathbf{0 . 0 4 5 5 4 9}$ & $\mathbf{3 . 4 1 7 7 4 5}$ & $\mathbf{0 . 1 0 0 8 5 0}$ & $\mathbf{5 . 2 0 6 4 4 6}$ \\
\% nominal & $\mathbf{1 . 6 0}$ & $\mathbf{1 . 2 6}$ & $\mathbf{3 . 6 6}$ & $\mathbf{1 . 9 7}$ \\
Ratio of means & $\mathbf{1 0 2 . 1 5}$ & $\mathbf{1 0 2 . 8 5}$ & $\mathbf{9 8 . 9 9}$ & $\mathbf{1 0 0 . 3 0}$ \\
\hline
\end{tabular}




\section{Acknowledgements}

Authors are thankful to Sir C R Reddy College of Pharmaceutical Sciences, Eluru, A.P, India, and Krishna University, Machilipatnam, A.P, India.

\section{Authors' contributions}

Research scholar: KNR. Research guides: Y.A. C and M. V.B.R. The authors have read and approved the manuscript.

\section{Funding}

No funding was received.

\section{Availability of data and materials}

All data and material are available upon request.

\section{Ethics approval and consent to participate}

Not applicable.

\section{Consent for publication}

Not applicable.

\section{Competing interests}

We declare(s) that we have no competing interests.

\section{Author details}

'Department of Pharmaceutical Chemistry, Krishna University, Krishna District, Machilipatnam, AP, India. ${ }^{2}$ Sir C. R Reddy College of Pharmaceutical Sciences, W.G.District, Eluru, AP, India. ${ }^{3}$ NRI College of Pharmacy, Pothavarappadu, Agiripalli, Rural, Krishna District, Vijayawada, AP, India. ${ }^{4}$ Department of Chemistry, Krishna University, Dr. MRAR Campus, Nuzvid 521201, India.

Received: 5 August 2020 Accepted: 7 December 2020

Published online: 22 January 2021

\section{References}

1. Gniadecki R, Assaf C, Bagot M, Dummer R, Duvic M, Knobler R (2007) The optimal use of bexarotene in cutaneous T-cell lymphoma. Br J Dermatol 157(3):433-440

2. van de Merbel NC, van Veen JH, Wilkens G, Loewen G (2002) Validated liquid chromatographic method for the determination of bexarotene in human plasma. J Chromatography B, Anal Technol Biomed Life Sci 775(2): 189-195

3. Rowe A (1997) Retinoid X receptors. Int J Biochem Cell Biol 29(2):275-278

4. Liyan Q, Tang X (2010) Bexarotene: a promising anticancer agent. Cancer Chemother Pharmacol 65(2):201-205

5. Dragnev KH, Petty WJ, Shah SJ, Lewis LD, Black CC, Memoli V, Nugent WC, Hermann T, Negro-Vilar A, Rigas JR, Dmitrovsky E (2007) A proof-of-principle clinical trial of bexarotene in patients with non-small cell lung cancer (PDF). Clinical cancer research. Clin Cancer Res 13(6):1794-1800

6. Esteva FJ, Glaspy J, Baidas S, Laufman L, Hutchins L, Dickler M, Tripathy D, Cohen R, DeMichele A, Yocum RC, Osborne CK, Hayes DF, Hortobagyi GN, Winer E, Demetri GD (2003) Multicenter phase II study of oral bexarotene for patients with metastatic breast cancer (PDF). J Clin Oncol 21(6):999-1006

7. ModelsCramer PE, Cirrito JR, Wesson DW, Lee CYD, Karlo JC, Zinn AE, Casali BT, Restivo JL (2012) ApoE-directed therapeutics rapidly clear $\beta$-amyloid and reverse deficits in AD mouse. Science 335(6075):1503-1506

8. Mogili R, Kanala K, Challa BR, Chandu BR, Bannoth CK (2011) Development and validation of amisulpride in human plasma by HPLC coupled with tandem MS and its application to a pharmacokinetic study. Sci Pharm 79(3): 583-599

9. Susantakumar P, Gaur A, Sharma P (2011) Development and validation of LC-MS/MS: method for the estimation acyclovir in pharmaceutical dosage form. Pharmacophore 2(4):173-187

10. Trivedi HK, Patel MC (2012) Development of stability indicating RPHPLC method for the determination of rupatadine and its degradation products in solid oral dosage form. Sci Pharm 80(4):889-902

11. Gajula R, Pilli NR, Ravi VB, Maddela R, Inamadugu JK, Polagani SR, Busa S (2012) Simultaneous determination of atorvastatin and aspirin in human plasma by LC-MS/MS: its pharmacokinetic application. Sci Pharm 80(4):923940
12. Selvadurai M, Meyyanathan SN (2011) Determination of deflazacort in human plasma by LC-MS after liquid-liquid extraction and its application in human pharmacokinetics studies. Pharmaceutical Methods 2(2):106-111

13. Huang L, Li X, Marzan F, Lizak PS, Aweeka FT (2012) Determination of lumefantrine in small-volume human plasma by LC-MS/MS: using a deuterated lumefantrine to overcome matrix effect and ionization saturation. Bioanalysis 4(2):157-166

14. Sharma VK, Glick J, Liao Q, Shen C, Vouros P (2012) GenoMass software: tool based on electrospray ionization tandem mass MS for characterization and sequencing of oligonucleotide adducts. J Mass Spectrum 47(4):490-501

15. Daniel CS (2010) Development and application of quality standard procedures for an LC-MS system Christy Sarmiento Daniel- Erasmus Mundus

16. Patel D (2011) Matrix effect in a view of LC-MS/MS: an overview. Int J Pharm Bio Sci 2(1):559-564

17. Targretin capsules - summary of product characteristics. (2013) electronic Medicines Compendium. Eisai Ltd.

\section{Publisher's Note}

Springer Nature remains neutral with regard to jurisdictional claims in published maps and institutional affiliations.

\section{Submit your manuscript to a SpringerOpen ${ }^{\circ}$ journal and benefit from:}

- Convenient online submission

- Rigorous peer review

- Open access: articles freely available online

- High visibility within the field

- Retaining the copyright to your article

Submit your next manuscript at $\boldsymbol{\nabla}$ springeropen.com 